\title{
Anemia materna y peso al nacer en productos de embarazos a término
}

\author{
José Ramón Urdaneta Machado, $P h D^{1}$, Marielis Lozada Reyes ${ }^{2}$, Maritza Cepeda de \\ Villalobos, $P h D^{3}$, José García I,PhD 2,3, Noren Villalobos I, PhD ${ }^{2,4}$, Alfi Contreras Benítez ${ }^{5}$, \\ Ana G. Ruíz, $P h D^{1}$, Olga Briceño Polacre, PhD. ${ }^{1}$ \\ ${ }^{1}$ Departamento de Morfofisiopatología, Facultad de Medicina. Universidad del Zulia. ${ }^{2}$ Programa de Especialización en \\ Obstetricia y Ginecología, Facultad de Medicina, Universidad del Zulia. ${ }^{3}$ Departamento de Obstetricia y Ginecología, \\ Facultad de Medicina, Universidad del Zulia. ${ }^{4}$ Cátedra de Anatomía, Facultad de Medicina, Universidad del Zulia. \\ ${ }^{5}$ Cátedra de Anatomía Humana, Facultad de Odontología, Universidad del Zulia. Venezuela.
}

\section{RESUMEN}

Objetivo: Relacionar la anemia materna con el peso al nacer (PAN) en mujeres con embarazos a término atendidas en la emergencia obstétrica de la Maternidad "Dr. Armando Castillo Plaza", Maracaibo, Venezuela. Métodos: Investigación correlacional con diseño no experimental y transeccional, donde se evaluaron 200 embarazadas en fase activa del trabajo de parto, a quienes se les determinaron los valores de hemoglobina $(\mathrm{Hb})$, hematocrito (Hcto) e índices hematimétricos, para luego correlacionarlas con el PAN. Resultados: Los valores de $\mathrm{Hb}$ oscilaban entre $8,4 \pm 1,0 \mathrm{~g} / \mathrm{dl}$ y $11,6 \pm 0,64 \mathrm{~g} / \mathrm{dl}$, mientras que los de Hcto fueron de $28,8 \pm 3,3 \%$ y $38,9 \pm 2,2 \%$, anémicas y no anémicas, respectivamente. Los índices hematimétricos mostraron valores referenciales normales en ambos grupos. El PAN de los recién nacidos de madres anémicas estaba disminuido en $12,39 \%(-420 \mathrm{~g})$ al compararse con los pesos de los neonatos de madre sin anemia $(2.970 \pm 0,43 \mathrm{~g}$ vs. $3.390 \pm 0,32 \mathrm{~g} ; \mathrm{p}<0,0001)$. El BPN fue más frecuente en el grupo de madres anémicas, las cuales mostraron un mayor riesgo, aunque no significativo (15\% vs. $10 \%$; OR IC $95 \% 1,558$ $[0,676$ - 3,728]; $p>0,05)$. Se demostró una relación directamente proporcional y significativa entre los valores de $\mathrm{Hb}$ - PAN $(r=0,439 ; p<0,0001)$. Conclusión: Existe una relación directa, proporcional y significativa entre el PAN y los valores de $\mathrm{Hb}$; sin embargo, aunque las gestantes anémicas presentaron con mayor frecuencia BPN, esta diferencia no fue significativa.

\section{PALABRAS CLAVE: Anemia, peso al nacer, embarazo a término}

\section{SUMMARY}

Aim: To link maternal anemia and birth weight (BW) in women with term pregnancies present to emergency obstetric at the Maternity "Dr. Armando Castillo Plaza", in Maracaibo, Venezuela. Methods: A correlational research, with non-experimental and transactional design, where valued a sample of 200 pregnant women in active phase of labor, who are determined hemoglobin $(\mathrm{Hb})$, hematocrit (Hct), and hematimetric indexes values. Results: The values of $\mathrm{Hb}$ and $\mathrm{Hct}$ in anemic ranged from $8.4 \pm 1 \mathrm{~g} / \mathrm{dl}$ and $11.6 \pm 0.64 \mathrm{~g} /$ $\mathrm{dl}$, whereas the Hct was $28.8 \pm 3.3 \%$ and $38.9 \pm 2.2 \%$, in patients with and without anemia, respectively. The hematimetric indexes showed normal reference values in both groups. The BW in newborn of anemic mothers was decreased by $12.39 \%(-420 \mathrm{~g})$ when compared to the weights of infants of mother without anemia $(2.970 \pm 0.43 \mathrm{~g}$ vs. $3.390 \pm 0.32 \mathrm{~g} ; \mathrm{p}<0.0001)$. LBW was more common in the group of anemic mothers, who showed an increased risk, although not significant $(15 \%$ vs. $10 \%$; OR $95 \% \mathrm{Cl} 1.588[0,676$ $3,728] ; p>0.05)$. Was demonstrated a directly proportional and significant relationship between $\mathrm{Hb}$ values 
and $B W(r=0.439 ; p<0.0001)$. Conclusion: Exist a direct proportional and significant relationship between the PAN and the $\mathrm{Hb}$; however, while anemic pregnant women presented more frequently LBW, this difference was not significant.

\section{KEY WORDS: Anemia, birth weight, term pregnancy}

\section{INTRODUCCIÓN}

La anemia y la ferropenia constituyen dos de los problemas nutricionales más frecuentes en la población especialmente en grupos vulnerables de países en vías de desarrollo (1). Las embarazadas constituyen uno de estos grupos por los altos requerimientos de hierro durante la gestación, que pueden representar hasta un tercio de los depósitos totales maternos $(2,3)$. De las casi 2.000 millones de personas anémicas estimadas en el mundo por la Organización Mundial de la Salud (OMS) (4), se estima que afecte a 77 millones de niños y mujeres en Latinoamérica y el Caribe (5).

La anemia se entiende como una condición en la cual los glóbulos rojos no están suministrando el oxígeno adecuado a los tejidos corporales, de la cual existen diversos tipos y causas (6), aunque en parte es debida a la hemodilución fisiológica que acontece durante este periodo, prevalecen la anemia ferropénica (50\% de los casos), megaloblástica y de células falciformes $(7,8)$. También puede deberse a otras deficiencias nutricionales (vitamina A), procesos infecciosos u inflamatorios $(9,10)$.

La prevalencia de anemia gestacional varía considerablemente, se sabe que afecta a casi la mitad de todas las embarazadas en el mundo: al $23 \%$ de las gestantes de países desarrollados y al $52 \%$ en naciones en vías de desarrollo; constituyendo un problema de salud pública que contribuye sustancialmente al aumento de la morbi-mortalidad materna y perinatal (11). Las gestantes con anemia durante los dos primeros trimestres, sobre todo ferropénicas, presentan un riesgo dos veces mayor para presentar parto pretérmino y hasta tres veces mayor para tener recién nacidos con bajo peso al nacer (BPN) (12).

En Venezuela la ferropenia y la anemia constituyen las carencias nutricionales más comunes, estimándose que hasta el $70 \%$ de su población femenina presenta déficit de hierro y más de un tercio de ella desarrolla anemia (13). En las embarazadas afecta en especial a las adolescentes y mujeres más pobres, con una prevalencia de $38 \%$ de anemia y $59 \%$ de deficiencia de hierro, por lo tanto es alto el riesgo de BPN, prematuridad y otras alteraciones producto de estas deficiencias (5).

El peso al nacer (PAN) es una variable clave para la morbilidad fetal y neonatal, que pronostica la supervivencia del neonato, su crecimiento, salud a largo plazo y desarrollo psicosocial (1416); por tanto la prevención del BPN es uno de los pilares para el desarrollo de la salud reproductiva y su reducción es una de las metas para reducir la mortalidad infantil en los objetivos del milenio de la declaración de Naciones Unidas $(17,18)$. En Venezuela, su prevalencia promedio es de $7,32 \%$, con una mortalidad perinatal de $32,49 \%$, fetal de $19,85 \%$ y neonatal de $12,63 \%$ (19); similar al $8,1 \%$ reportada en Latinoamérica (20) y al 7\% señalado en Estados Unidos (21).

Existen diversos factores o afecciones que pueden acompañar a la gestación e interferir con la nutrición fetal y con su ganancia de peso. Estudios previos han señalado que el PAN puede ser influenciado por la presencia de anemia, encontrándose una asociación entre los niveles bajos de $\mathrm{Hb}$ y el BPN (22-30). Por tanto, surgió la necesidad de relacionar la anemia materna y el PAN en pacientes con embarazos a término.

\section{PACIENTES Y MÉTODOS}

Investigación correlacional con diseño no experimental y transeccional, donde se evaluó una muestra intencionada conformada por 200 mujeres con embarazos a término que acudieron a la emergencia obstétrica de la Maternidad "Dr. Armando Castillo Plaza", Maracaibo, estado Zulia, Venezuela. Se incluyeron pacientes a término, en fase activa del trabajo de parto, índice de masa corporal mayor de $15 \mathrm{Kg} / \mathrm{m} 2$ y menor a $25 \mathrm{Kg} / \mathrm{m} 2$, que manifestaron haber recibido suplementación de hierro durante el embarazo y que asistieron a control prenatal en la institución ámbito del estudio. Se excluye gestantes con embarazo pretérmino, postérmino o gemelares, gestaciones complicadas o asociadas a patologías crónicas, adolescentes ( $<19$ años), pacientes con óbito fetal o diagnóstico prenatal de malformaciones congénitas.

Las pacientes participaron voluntariamente y previa explicación del propósito y procedimiento de la investigación, les fue solicitado su consentimiento informado por escrito. El estudio planteado no representaba riesgo para las embarazadas ni para sus fetos o recién nacidos, respetando las normas de la declaración de Helsinki. El protocolo de estudio fue previamente aprobado por el Comité de Bioética de la Institución.

A cada participante, se les tomó mediante 
acceso venoso periférico tres (3) mililitros de sangre de la vena antecubital, los cuales fueron introducidos en un tubo de polietileno con EDTA (Vacutainer()). Para determinar la biometría hemática y los índices eritrocitarios se utilizó un contador de células automatizado Marca Sysmex F-500 para procesar muestras sanguíneas y la concentración de $\mathrm{Hb}$ se estableció por el método de cianmetahemoglobina. El diagnóstico de anemia se realizó según los criterios de la OMS (4): las embarazadas con $\mathrm{Hb}$ inferior a $11 \mathrm{~g} / \mathrm{dl}$ y Hcto menor a $33 \%$ se consideraron anémicas y conformaron el grupo "A" $(n=100)$, las pacientes con valores igual o superior conformaron el grupo "B" o no anémicas $(n=100)$.

Posterior al parto e independientemente la vía del nacimiento, se procedió a registrar el PAN de acuerdo al sexo de los recién nacidos; para ello todos fueron pesados inmediatamente en una bascula digital, perfectamente calibrada, en posición decúbito dorsal y completamente desnudos. El peso obtenido fue expresado en gramos y la edad gestacional determinada por el método de Capurro. Las alteraciones en el PAN fueron definidas de acuerdo con los criterios de la OMS (31), como BPN aquellos neonatos con peso menor a 2.500 gramos o macrosomía si pesaban más de 4.000 gramos.

Los datos obtenidos fueron analizados mediante el Paquete Estadístico para Ciencias Sociales (SPSS), versión 19; ejecutándose un tratamiento estadístico descriptivo donde los datos fueron expresados mediante frecuencias absolutas o relativas (porcentajes), medidas de tendencia central (medias) y de dispersión (desviación estándar). La relación entre los parámetros hematimétricos maternos con el PAN se determinó mediante el coeficiente de correlación de Pearson y para la comparación entre grupos se utilizaron las pruebas t de Student o del
Chi cuadrado, con un nivel de confianza del 95\% y significación estadística de $p<0,05$.

\section{RESULTADOS}

Entre los grupos de gestantes con y sin anemia, no hubo diferencias significativas en cuanto a la edad, edad gestacional e índice de masa corporal (IMC) pre-concepcional; en las pacientes con anemia hubo una menor ganancia de peso en el embarazo y la paridad mayor $(p<0,001)$ (Tabla I).

En cuanto a los aspectos socioeconómico (Tabla II) entre anémicas y no anémicas, se observó que la totalidad de la muestra corresponde a mujeres en situación de pobreza, predominando el concubinato como estado civil (43\% y $46 \%$, respectivamente), grado de instrucción secundaria incompleta (33\% y $32 \%$, respectivamente), tipo de vivienda de interés social ( $48 \%$ y $64 \%$, respectivamente) e ingreso familiar con sueldos iguales al sueldo mínimo ( $76 \%$ y $72 \%$ respectivamente).

La Tabla III, presenta los parámetros hematológicos promedio de ambos grupos. Los valores de $\mathrm{Hb}$ y Hcto promedio en las embarazadas anémicas fue de $8,4 \pm 1 \mathrm{~g} / \mathrm{dl}$ y $28,8 \pm 3,3 \%$; en las gestantes sin anemia, fue de $11,6 \pm 0,64 \mathrm{~g} / \mathrm{dl}$ y $38,9 \pm 2,2 \%$. Los índices hematimétricos: volumen corpuscular medio (VCM), concentración de hemoglobina corpuscular media (CHCM) y concentración hemática media (HCM), se ubicaron entre los valores referenciales normales en ambos grupos. Hubo diferencias significativas entre ambos grupos, en todos los parámetros evaluados. Con respecto a la severidad de la anemia, al clasificar el grado de anemia en las 100 pacientes estudiadas del grupo "A" se evidenció un predominio de los casos de anemia moderada $(87 \%)$ y en menor frecuencia la severa $(13 \%)$, no hubo casos de anemia leve.

\section{Tabla I
CARACTERIZACIÓN DE LA MUESTRA DE GESTANTES A TÉRMINO EN TRABAJO DE PARTO CON Y SIN ANEMIA}

\begin{tabular}{lccc}
\hline Características & $\begin{array}{c}\text { Grupo “A" } \\
\text { (Anémicas) }\end{array}$ & $\begin{array}{c}\text { Grupo “B” } \\
\text { (No Anémicas) }\end{array}$ & $\mathrm{p}^{*}$ \\
\cline { 2 - 3 } & Media $\pm \mathrm{DE}$ & Media $\pm \mathrm{DE}$ & \\
\hline Edad (años) & $24,7 \pm 4,3$ & $24,4 \pm 4,5$ & 0,630 \\
Edad Gestacional (semanas) & $38,4 \pm 1,3$ & $38,4 \pm 0,7$ & 1,000 \\
IMC pre-concepcional (kg/m2) & $20,1 \pm 3,2$ & $20,6 \pm 3,4$ & 0,285 \\
Ganancia ponderal en el embarazo $(\mathrm{kg})$ & $9,4 \pm 1,7$ & $11,8 \pm 4,4$ & 0,000 \\
Paridad & $2,9 \pm 1,9$ & $2,1 \pm 1,2$ & 0,000 \\
\hline
\end{tabular}

$\mathrm{DE}=$ Desviación estándar

$\mathrm{n}=100$ para cada grupo

* Prueba T de Student. Nivel de significancia $p<0,05$ 


\section{DATOS SOCIOECONÓMICOS DE LAS GESTANTES A TÉRMINO EN TRABAJO DE PARTO CON Y SIN ANEMIA}

\begin{tabular}{|c|c|c|c|c|}
\hline \multirow[t]{2}{*}{ Datos socioeconómicos } & \multicolumn{2}{|c|}{$\begin{array}{l}\text { Grupo "A" } \\
\text { (Anémicas) }\end{array}$} & \multicolumn{2}{|c|}{$\begin{array}{c}\text { Grupo “B” } \\
\text { (No Anémicas) }\end{array}$} \\
\hline & $\mathrm{n}$ & $\%$ & $\mathrm{n}$ & $\%$ \\
\hline \multicolumn{5}{|l|}{ Estado civil } \\
\hline - Soltera & 18 & 18 & 18 & 18 \\
\hline - Casada & 39 & 39 & 36 & 36 \\
\hline - Concubina & 43 & 43 & 46 & 46 \\
\hline \multicolumn{5}{|l|}{ Grado de instrucción } \\
\hline - Primaria incompleta & 3 & 3 & 1 & 1 \\
\hline - Primaria completa & 15 & 15 & 13 & 13 \\
\hline - Secundaria incompleta & 33 & 33 & 32 & 32 \\
\hline - Secundaria completa & 31 & 31 & 22 & 22 \\
\hline - Técnico & 13 & 13 & 20 & 20 \\
\hline - Universitario & 5 & 5 & 12 & 12 \\
\hline \multicolumn{5}{|l|}{ Tipo de vivienda } \\
\hline - Rancho & 10 & 10 & 4 & 4 \\
\hline - Vivienda de interés social & 48 & 48 & 64 & 64 \\
\hline - Casa/quinta & 38 & 38 & 14 & 14 \\
\hline - Apartamento & 4 & 4 & 18 & 18 \\
\hline \multicolumn{5}{|l|}{ Ingreso familiar } \\
\hline - < Sueldo mínimo & 22 & 22 & 24 & 24 \\
\hline - Sueldo mínimo & 76 & 76 & 72 & 72 \\
\hline - > Sueldo mínimo & 2 & 2 & 4 & 4 \\
\hline
\end{tabular}

$\mathrm{n}=100$ para cada grupo

Tabla III

PARÁMETROS HEMATIMÉTRICOS EN GESTANTES A TÉRMINO EN TRABAJO DE PARTO CONY SIN ANEMIA

\begin{tabular}{lccc}
\hline Parámetros & $\begin{array}{c}\text { Grupo "A" } \\
\text { (Anémicas) }\end{array}$ & $\begin{array}{c}\text { Grupo "B" } \\
\text { (No Anémicas) }\end{array}$ & $\mathrm{p}^{*}$ \\
\cline { 2 - 4 } Media $\pm \mathrm{DE}$ & Media \pm DE & $11,6 \pm 0,6$ & 0,000 \\
Hemoglobina (g/dl) & $8,4 \pm 1,0$ & $38,9 \pm 2,2$ & 0,000 \\
Hematocrito (\%) & $28,9 \pm 3,3$ & $92,2 \pm 19,7$ & 0,000 \\
VCM (fl) & $80,7 \pm 11,4$ & $29,0 \pm 2,7$ & 0,042 \\
CHCM (\%) & $28,6 \pm 2,3$ & $28,3 \pm 4,0$ & 0,000 \\
HCM (\%) & $23,9 \pm 3,8$ & & \\
\hline
\end{tabular}

$\mathrm{DE}=$ Desviación estándar.

$\mathrm{n}=100$ para cada grupo.

* Prueba T de Student. Nivel de significancia $p<0,05$ 
En cuanto a los recién nacidos de las gestantes a término en trabajo de parto incluidas en el estudio (Tabla IV), en el grupo de madres anémicas se caracterizaron por ser predominante del sexo masculino $(54 \%)$ y con una edad gestacional media de $38,5 \pm 0,61$ semanas determinadas por el método de Capurro; en tanto que en las mujeres sin anemia, fueron en su mayoría del sexo femenino (68\%) y con una edad gestacional similar $(38,6 \pm 0,65)$. Cuando se indaga la variable de interés (PAN), se observa que el peso medio de los recién nacidos de madres anémicas es significativamente menor $(2.970 \pm 0,43 \mathrm{~g})$ en comparación a los neonatos de madre no anémicas $(3 \cdot 390 \pm 0,32 \mathrm{~g})(\mathrm{p}<0,0001)$. El BPN predominó en las madres anémicas (15\% vs. $10 \%$ ), diferencia no significativa (OR [IC95\%]= 1,558 [0,676 - 3,728]; p>0,05) (Tabla V).

Al relacionar el PAN con cada uno de los parámetros hematológicos, se pudo demostrar que existe una asociación directa y significativa entre las variables $\mathrm{Hb}-\operatorname{PAN}(r=0,439 ; \mathrm{p}=0,000)$, Hcto - PAN $(r=0,426 ; p=0,000)$ y VCM - PAN $(r=0,209$; $\mathrm{p}=0,011)$; mientras que en los demás índices hematimétricos no se evidenció asociación con el PAN (Tabla VI).

\section{DISCUSIÓN}

Los resultados presentados en este estudio demostraron que existía una diferencia altamente significativa entre el PAN de los neonatos de madres con y sin anemia $(p<0,0001)$, con una relación directamente proporcional entre el PAN y los valores de $\mathrm{Hb}$ y Hcto $(\mathrm{p}<0,0001$; respectivamente); aunque los recién nacidos de las gestantes anémicas presentaron una mayor prevalencia de BPN, esta diferencia no resultó ser significativa $(p>0,05)$. En cuanto a los índices hematimétricos, su relación fue baja, quizás porque dichos parámetros permite

Tabla IV

ANÁLISIS DESCRIPTIVO DEL PESO AL NACER EN RECIÉN NACIDOS PRODUCTO DE GESTANTES A TÉRMINO CON Y SIN ANEMIA

\begin{tabular}{lc}
\hline Grupo & $\begin{array}{c}\text { Peso (g) al nacer * } \\
\text { (Media } \pm \text { DE) }\end{array}$ \\
\hline $\begin{array}{l}\text { Grupo "A" } \\
\text { (Anémicas) }\end{array}$ & $2.970 \pm 0,43$ \\
$\begin{array}{l}\text { Grupo "B" } \\
\text { (No Anémicas) }\end{array}$ & $3.390 \pm 0,32$ \\
\hline
\end{tabular}

$\mathrm{DE}=$ Desviación estándar.

$\mathrm{n}=100$ para cada grupo.

${ }^{*} p<0,0001$ (Comparación por $\mathrm{T}$ de student; $t=7,835$; df 198).

\section{Tabla V}

CLASIFICACIÓN DEL PESO AL NACER EN RECIÉN NACIDOS PRODUCTO DE GESTANTES A TÉRMINO CONY SIN ANEMIA

\begin{tabular}{lcccc}
\hline Clasificación & \multicolumn{2}{c}{ Grupo "A" } & \multicolumn{2}{c}{ Grupo "B" } \\
& \multicolumn{2}{c}{ (Anémicas) } & \multicolumn{2}{c}{ (No Anémicas) } \\
& $\mathrm{n}$ & $\%$ & $\mathrm{n}$ & $\%$ \\
\hline $\begin{array}{l}\text { Bajo peso al nacer * } \\
(<2.500 \mathrm{~g})\end{array}$ & 15 & 15 & 10 & 10 \\
$\begin{array}{l}\text { Peso adecuado } \\
(2.501-3.999 \mathrm{~g})\end{array}$ & 85 & 85 & 90 & 90 \\
\hline
\end{tabular}

$\mathrm{n}=100$ para cada grupo

* OR [IC95\%]= 1,588 [0,676 - 3,728] (Análisis de regresión logística)

$\mathrm{p}=0,392\left(x^{2}=0,731\right)$

Tabla VI

RELACIÓN DEL PESO AL NACER CON LOS PARÁMETROS HEMATOLÓGICOS

DE GESTANTES A TÉRMINO EN TRABAJO DE PARTO CON Y SIN ANEMIA

\begin{tabular}{|c|c|c|}
\hline Parámetros $\quad$ Peso al nacer & $r$ & $p^{*}$ \\
\hline Hemoglobina & 0,439 & 0,000 \\
\hline Hematocrito & 0,426 & 0,000 \\
\hline VCM & 0,209 & 0,011 \\
\hline $\mathrm{CHCM}$ & 0,048 & 0,560 \\
\hline $\mathrm{HCM}$ & 0,159 & 0,053 \\
\hline
\end{tabular}

* Correlación bivariada de Pearson.

La correlación es significativa al nivel de 0,01 (bilateral).

evaluar en el glóbulo rojo tamaño y forma, asociado más a anemias no nutricionales. Es necesario destacar que la anemia nutricional, es frecuente en el embarazo, producto del déficit de micronutrientes y afectan principalmente los valores de $\mathrm{Hb}$ y Hcto (10).

Estos resultados fueron similares a los reportados por Yildiz y cols (23), quienes evidenciaron que los valores bajos de $\mathrm{Hb}$ durante el tercer trimestre de gestación se asociaron tanto con la talla de los recién nacidos $(p<0,001)$ como con el BPN; asociándose una concentración de $\mathrm{Hb}$ alta durante el tercer trimestre con un mayor PAN (OR [IC95\%]= $1,08[01,05-01,11] ; p<0,001)$ y una correlación positiva y significativa entre las cifras de $\mathrm{Hb}$ con el PAN. 
De igual manera, Ma y cols (32), encontraron que los niveles maternos bajos de $\mathrm{Hb}$ tenían influencia en el PAN, sobre todo con valores de $\mathrm{Hb}$ inferiores a $8 \mathrm{~g} / \mathrm{dl}$; en tanto que Lelic y cols (28), encontraron que los neonatos de madres anémicas tenían significativamente menor $(\mathrm{p}<0.0001)$ la menor talla $y$ el PAN $(51,76$ vs. $55,54 \mathrm{~cm}$ y $3.048,00 \mathrm{~g}$ vs. 3615,60 g). También, en mujeres colombianas se ha evidenciado que la concentración de $\mathrm{Hb}$ era menor en las madres con bajo IMC y PAN, correlacionándose positivamente con este último indicador (1).

Contrario a los resultados encontrados en este investigación, así como en la mayor parte de los trabajos revisados, un estudio efectuado por Mardones y cols (2), no encontró una diferencia significativa entre el peso fetal y anemia $(p=0,966)$; determinando que la anemia al inicio del embarazo no se asociaba con el crecimiento fetal. Igualmente, otros autores (33) determinaron que los valores elevados de $\mathrm{Hb}$, más no la anemia, era lo que se asociaba significativamente con una restricción del peso fetal desde el tercer trimestre $(p<0,05)$; mientras que en un estudio brasileño realizado en adolescentes embarazadas no se constató una asociación significativamente entre la anemia y PAN (34).

Por otra parte, Demmouche y cols (35) determinaron una correlación positiva y directamente proporcional entre el nivel de $\mathrm{Hb}$ y el PAN $(r=0,28)$, aunque no significativa ( $p>0,05)$; sin evidenciarse diferencias estadísticamente significativas entre el promedio del PAN y los diversos valores de $\mathrm{Hb}$, lo cual sugiere que otros parámetros, diferentes a la concentración de $\mathrm{Hb}$ materna, pudiesen influir en el PAN. En este sentido, otras investigaciones han encontrado asociación significativa entre el PAN y las reservas de ferritina, teniendo las gestantes con depleción de hierro (ferritina sérica <12 $\mu \mathrm{g} / \mathrm{l}$ ) al inicio del embarazo neonatos con un promedio de 192 gramos menos $(p=0,028)$ que aquellas con reservas de hierro normales (36).

Respecto al BPN, a pesar que diversos estudios han reconocido a la anemia como un factor de riesgo independiente para su presentación (23-30); en esta investigación, aunque se evidenció una mayor prevalencia en el grupo de gestantes anémicas, esta diferencia no fue significativa $(p>0,05)$. No obstante, la prevalencia de neonatos con BPN, fue mucho menor que la reportada en otro estudio venezolano donde se encontró anemia materna en el $40,6 \%$ de los casos de BPN (37).

A diferencia de los resultados presentados en esta serie, un estudio hindú (38) determinó una prevalencia elevada y significativa $(p=0,000)$ de recién nacidos con BPN tanto en casos de anemia leve $(20,3 \%)$ como moderada/severa $(24,6 \%)$, al compararlas con las no anémicas (19,6\%). De igual manera, otros investigadores revelaron que $28,8 \%$ de los casos de BPN se asociaban con anemia gestacional (26); mientras que en una investigación realizada en Cuba (27) el $76 \%$ de las mujeres que tenía anemia durante el tercer trimestre del embarazo tuvieron hijos con BPN.

En cuanto al riesgo de presentar un neonato con BPN, las embarazadas anémicas evaluadas en este estudio presentaron un incremento no significativo de casi el doble en el riesgo para tener un producto con BPN. Sin embargo, otros autores han podido establecer que la anemia se asocia a un riesgo significativo de presentar neonatos con BPN. Al respecto, en una investigación cubana (27) se determinó un riesgo significativo de 7 veces más probabilidades de tener neonatos con BPN que las que tienen cifras de $\mathrm{Hb}$ superiores a $110 \mathrm{~g} / \mathrm{l}$ (OR $[\mathrm{IC} 95 \%]=7,39$ [3,19-17,40]; $\mathrm{p}<0,001)$. Asimismo, en mujeres chinas (26) se obtuvo una probabilidad cinco veces mayor (OR [IC95\%]= 5,03 [4,325 5,853]; $p<0,001)$; mientras que en mujeres hindúes se reportó el doble del riesgo (OR [IC95\%]= 2,3 [1,08 - 5,18]) (25).

Aunque, Kumar y cols (39) también evidenciaron que el PAN de los neonatos de madres anémicas fue inferior al de los de madres no anémicas (2,88 vs 3,04 kg; $p<0,05)$; a diferencia de esta investigación, ellos sí pudieron demostrar un incremento significativo en la incidencia de BPN en gestantes con anemia durante el tercer trimestre al compararlas con embarazadas no anémicas (14,31\% vs. $7,80 ; p=0,001)$. Asimismo, otro estudio detectó que valores de $\mathrm{Hb}$ inferiores a $10 \mathrm{~g} / \mathrm{dl}$ se asociaban con un aumento significativo del riesgo de BPN; aunque cifras mayores a $13 \mathrm{~g} / \mathrm{dl}$ también representaron un riesgo, pero no significativo (40).

Se ha señalado que el riesgo de BPN depende de la severidad de la anemia, reportándose en las gestantes con anemia moderada/severa un riesgo significativo de tener un recién nacido con BPN $(28,29)$ e inclusive en los casos de anemia leve (29). En este sentido, otra investigación determinó que concentraciones bajas de $\mathrm{Hb}$ en el primer trimestre de la gestación parecen estar asociadas con un BPN, puesto que se observó una correlación positiva y significativa entre el peso fetal y un aumento de la concentración de $\mathrm{Hb}$ materna en el primer trimestre; aunque en el segundo y tercer trimestre no encontraron una correlación significativa entre el PAN y las concentraciones de $\mathrm{Hb}$ (24).

Estos hallazgos reflejan que la anemia puede causar directamente un pobre crecimiento fetal intrauterino debido al flujo inadecuado de oxígeno a los tejidos de la placenta o puede ser un indicador indirecto de la deficiencia de la nutrición materna $(23,24,41,42)$. Por tanto, la suplementación profiláctica prenatal con hierro es una medida útil para mejorar el PAN y potencialmente reducir los costos de salud, puesto que se ha demostrado que conduce a un incremento significativo en el promedio del PAN y a una disminución de neonatos con BPN (43-48). 
Actualmente la magnitud de la anemia sigue siendo de importancia alta, por lo que es necesario diseñar estrategias de prevención de la deficiencia de hierro, identificar y suplementar a las mujeres en fase de mayor riesgo de anemia y detectar tempranamente la anemia en las gestantes con el propósito de detener el desarrollo intergeneracional de la anemia y sus consecuencias asociadas (49). Cabe resaltar que Venezuela en la 65a Asamblea Mundial de la Salud realizada por la OMS se comprometió a cumplir 6 metas globales para lograr mejorías hasta el año 2025 en cuanto a la nutrición materna, del lactante y del niño pequeño; que incluían la reducción relativa del $50 \%$ de las tasas de anemia en mujeres en edad reproductiva y la reducción relativa del $30 \%$ en el número de niños nacidos con BPN (50).

Ante este compromiso y los resultados presentados, se hace necesario promover el consumo de alimentos biodisponibles en hierro mediante la educación nutricional, difundir información sobre las consecuencias en la salud que conlleva la anemia y la deficiencia de hierro, mejorar la atención prenatal y la suplementación de hierro u otros micronutrientes; adicionalmente promover y apoyar la lactancia materna exclusiva para mantener las reservas corporales de hierro después del parto, manteniendo el binomio lactancia-amenorrea en aquellas mujeres no anémicas o la suplementación con hierro en las anémicas.

Sin embargo, la intencionalidad de la muestra analizada y el número de sujetos que la conformaron constituyen una limitante para que estos resultados puedan generalizarse al resto del universo de estudio. De igual manera, dada la crisis hospitalaria que atraviesa el sistema de salud pública venezolano, aunada a la problemática inflacionaria y a la escasez de reactivos, se presentaron limitaciones para analizar otros parámetros que pudiesen afectar en el metabolismo férrico; además de indagar sobre la deficiencia de micronutrientes como causa de la anemia.

\section{CONCLUSIÓN}

Existe una asociación directa, proporcional y significativa entre el PAN y los valores de $\mathrm{Hb}(\mathrm{r}=$ 0,439; $p<0,0001$ ); lo cual demuestra la repercusión de la anemia materna sobre esta variable. Sin embargo, aunque en las gestantes anémicas el PAN fue significativamente menor $(p<0,0001)$ y presentaron una mayor frecuencia de BPN; esta ultima diferencia no fue significativa.

\section{REFERENCIAS}

1. Parra BE, Restrepo SL; Manjarrés LM, Mancilla LP. Indicadores bioquímicos del hierro materno en el tercer trimestre de la gestación y su relación con la antropometría materna y el peso al nacer. latreia. 2009; 22(1):16-26.

2. Mardones F, Duran E, Villarroel L, Gattini D, Ahumada D, Oyarzún F. et al. Anemia del embarazo en la Provincia de Concepción, Chile: relación con el estado nutricional materno y el crecimiento fetal. Arch Latinoam Nutr. 2008;58(2):132-138.

3. Rached I, Azuaje A, Henríquez G. (2002). Cambios en las variables hematológicas y bioquímicas durante la gestación en mujeres eutróficas. En: Anales Venezolanos de Nutrición 2002;15(1):11-7.

4. Organización Mundial de la Salud. La anemia como centro de atención. 2005. Disponible en: http://www. paho.org/Spanish/AD/FCH/NU/OMS04_Anemia.pdf. Acceso: 29 de Octubre de 2014.

5. Landaeta M, García M, Bosch V. Principales deficiencias de micronutrientes en Venezuela. Rev Esp Nutr Com. 2003;9 (3):117-27.

6. Naveda MJ, López J, Miller A. Repercusión de la anemia materna sobre el pico de velocidad sistólica de la arteria cerebral media fetal. Rev Obstet Ginecol Venez. 2010;70(4):224-32.

7. Romero M, Cuenca E, López G, Rosales C. Anemia y control del peso en embarazadas. Revista de Posgrado de la VI a Cátedra de Medicina. 2005;143:6-8.

8. Álvarez LC, Barros J, Ladrón M. Anemia aplástica y embarazo. Salud Uninorte. 2007;23(1):120-6.

9. Olivares $\mathrm{M}$, Walter $\mathrm{T}$. Consecuencias de la deficiencia de hierro. Rev Chil Nutr. 2003;30(3):226-33.

10. Urdaneta JR, Sánchez K, Cepeda M, Garcia J, Briceño $\mathrm{O}$, Baabel N, et al. Anemias nutricionales en gestantes adolescentes y adultas. MedULA. 2013;22(2):807.

11. Candio F, Hofmeyr G. Tratamientos para la anemia ferropénica en el embarazo: Comentario de la BSR. La Biblioteca de Salud Reproductiva de la OMS; Ginebra: Organización Mundial de la Salud. 2007.

12. Canaval H, Pérez H, Rincón D, Vargas J. Farmacología del Hierro. 1ra edición. Anemia Working Group Latin America (AWGLA), Colombia. 2009.

13. Cedillo N, Dellán J, Toro J. Estado nutricional de las adolescentes embarazadas: relación con el crecimiento fetal. Rev Obstet Ginecol Venez. 2006;66(4):233-40.

14. Torloni MR, Sass N, Leiko J, Pinheiro AC, Fukuyama $M$, de Lucca PR. Clinical formulas, mother's opinion and ultrasound in predicting birth weight. Sao Paulo Med J. 2008;126(3):145-9.

15. Pang MW, Leung TN, Lau TK. A validation study of ultrasonic foetal weight estimation models for Hong Kong Chinese singleton pregnancies. Hong Kong Med . 2004;10(6):384-8.

16. Valenti LL. Peso al nacer y su relación con síndrome metabólico en el adulto. Rev Soc Arg Diabetes. 2008;2(3):185-201.

17. Leal MC. Comportamiento del bajo peso al nacer y repercusión sobre la mortalidad infantil en el quinquenio 2001-2005. Rev Cub Obstet Ginecol. 2009;5(4):99107.

18. Daza V, Jurado W, Duarte D, Gich I, Sierra CH, Delgado $M$. Bajo peso al nacer: exploración de algunos factores de riesgo en el Hospital Universitario de San José en Popayán (Colombia). Rev Col Obstet Ginecol. 2009;60(2):124-34. 
19. Faneite P, Rivera C, Amato R, Faneite J, Paradas M. ¿Tiene importancia el bajo peso neonatal? Rev Obstet Ginecol Venez. 2011;71(3):151-7.

20. Organización Panamericana de la Salud. Health situation in the Americas. Basic Indicators 2009. Disponible en: http://new.paho.org/hq/index.php?option=com_co ntent\&task=view\&id $=1878 \&$ Itemid $=1723$. Acceso: 29 de Noviembre de 2014.

21. Glass R. Bajo peso al nacer. JAMA. 2002;287(2):1-2.

22. Cecatti JG, Medeiros CS. Correlação entre peso fetal estimado por ultrassonografia e peso neonatal. Ver Bras Ginecol Obstet. 2010;32(1):4-10.

23. Yildiz Y, Özgü E, Unlu SB, Salman B, Eyi EG. The relationship between third trimester maternal hemoglobin and birth weight/length; results from the tertiary center in Turkey. J Matern Fetal Neonatal Med. 2014;27(7):729-32.

24. Bakacak M, Avci F, Ercan O, Köstü B, Serin S, Kiran $\mathrm{G}$, et al. The effect of maternal hemoglobin concentration on fetal birth weight according to trimesters. J Matern Fetal Neonatal Med. 2014;27(11):1-5.

25. Kattula D, Sarkar R, Sivarathinaswamy P, Velusamy V, Venugopal S, Naumova EN, et al. The first 1000 days of life: prenatal and postnatal risk factors for morbidity and growth in a birth cohort in southern India. BMJ Open. 2014;4(7): e005404. Disponible en: http:www.ncbi.nlm.nih.gov/pmc/articles/ PMC4120427/pdf/bmjopen-2014005404.pdf. Acceso: 1 de Diciembre de 2014.

26. Chen Y, Li G, Ruan Y, Zou L, Wang X, Zhang W. An epidemiological survey on low birth weight infants in China and analysis of outcomes of full-term low birth weight infants. BMC Pregnancy Childbirth. 2013;13:242. Disponible en: http://www.biomedcentral.com/1471-2393/13/242. Acceso: 1 de Diciembre de 2014.

27. Rey A, Ávila D, Rodríguez A, Cabrera AC. Factores nutricionales maternos y el bajo peso al nacer en un área de salud. Revista Finlay. 2013;3(1). Disponible en: http://www.revfinlay.sld.cu/index.php/finlay/article/ view/172. Acceso: 29 de Noviembre de 2014.

28. Lelic M, Bogdanovic G, Ramic S, Brkicevic E. Influence of maternal anemia during pregnancy on placenta and newborns. Med Arh. 2014;68 (3):184-7.

29. Yi SW, Han YJ, Ohrr H. Anemia before pregnancy and risk of preterm birth, low birth weight and smallfor-gestational-age birth in Korean women. Eur J Clin Nutr. 2013;67(4):337-342.

30. O'Farrill F, O'Farrill M, Fragoso LE. Evaluación del tratamiento a mujeres embarazadas con anemia ferropénica. Ginecol Obstet Mex. 2013;81(7):377-81.

31. World Health Organization. Report of a WHO Expert Comitee. Physical status: The use and interpretation of anthropometry. Geneva: WHO; TRS 854; 1995.

32. Ma AG, Schouten E, Wang Y, Xu RX, Zheng MC, Li $Y$, et al. Anemia prevalence among pregnant women and birth weight in five areas in China. Med Princ Pract. 2009; 18:368-372. Disponible en: http://www. karger.com/mpp. Acceso: 25 de Septiembre de 2011.

33. Gaillard R, Eilers PH, Yassine S, Hofman A, Steegers EA, Jaddoe VW. Risk factors and consequences of maternal anaemia and elevated haemoglobin levels during pregnancy: a population-based prospective cohort study. Paediatr Perinat Epidemiol. 2014;28(3):213-26.

34. Surita FGC, Suarez MBB, Siani S, Silva JLP. Fatores associados ao baixo peso ao nascimento entre adolescentes no Sudeste do Brasil. Rev Bras Ginecol Obstet. 2011;33(10):286-91.

35. Demmouche A, Lazrag A, Moulessehoul S. Prevalence of anaemia in pregnant women during the last trimester: consequense for birth weight. Eur Rev Med Pharmacol Sci. 2011;15(4):436-45.

36. Ribot B, Aranda N, Viteri F, Hernández C, Canals J, Arija V. Depleted iron stores without anaemia early in pregnancy carries increased risk of lower birthweight even when supplemented daily with moderate iron. Hum Reprod. 2012;27(5):1260-6.

37. González Y, Álvarez A. Comportamiento del bajo peso al nacer en la parroquia "Castor Nieves Ríos. Venezuela". Rev Cubana Med Gen Integr. 2010;26(3):5-7.

38. Kader M, Perera NK. Socio-economic and nutritional determinants of low birth weight in India. $\mathrm{N} \mathrm{Am} \mathrm{J} \mathrm{Med}$ Sci. 2014;6(7):302-8.

39. Kumar KJ, Asha N, Murthy DS, Sujatha MS, Manjunath VG Maternal anemia in various trimesters and its effect on newborn weight and maturity: an observational study. Int J Prev Med. 2013;4(2):193-9.

40. Sekhavat L, Davar R, Hosseinidezoki S. Relationship between maternal hemoglobin concentration and neonatal birth weight. Hematology. 2011;16(6):373-6.

41. Rached I, Henríquez G, Azuaje A. Relación del peso al nacer con la edad gestacional y la antropometría materna. Anales Venezolanos de Nutrición. 2006;19(1):10-16.

42. Guerra M, García J, Labarca N, Cepeda M, Reyna E, Gómez M, Martínez O. Apgar y variables hematológicas en pacientes con anemia materna crónica severa y trabajo de parto normal. Rev Obstet Ginecol Venez. 2008;68(1):5-11.

43. Haider BA, Olofin I, Wang M, Spiegelman D, Ezzati M, Fawzi WW, et al. Anaemia, prenatal iron use, and risk of adverse pregnancy outcomes: systematic review and meta-analysis. BMJ. 2013;346:f3443. Disponible en: http://www.bmj.com/content/bmj/346/bmj.f3443. full.pdf. Acceso: 1 de Diciembre de 2014.

44. Balarajan Y, Subramanian SV, Fawzi WW. Maternal iron and folic acid supplementation is associated with lower risk of low birth weight in India. J Nutr. 2013;143(8):1309-15.

45. Wise J. Daily iron during pregnancy improves birth weight. BMJ. 2013;346:f3997. Disponible en: http:// www.bmj.com/content/346/bmj.f3997.full.pdf+html. Acceso: 1 de Diciembre de 2014.

46. Imdad A, Bhutta ZA. Routine iron/folate supplementation during pregnancy: effect on maternal anaemia and birth outcomes. Paediatr Perinat Epidemiol. 2012;26(Suppl 1):168-77.

47. Cogswell ME, Parvanta I, Ickes L, Yip R, Brittenham GM. Iron supplementation during pregnancy, anemia, and birth weight: a randomized controlled trial. Am J Clin Nutr. 2003;78(4):773-81.

48. Saha L, Pandhi P, Gopalan S, Malhotra S, Saha PK. Comparación de eficacia, tolerancia y coste del 
complejo de hierro polimaltosato frente al sulfato ferroso (SF) en el tratamiento de la anemia por deficiencia de hierro en mujeres embarazadas. Medscape General Medicine. 2007;9(1):1.

49. Chaparro C, Lutter Ch. La anemia entre adolescentes y mujeres adultas jóvenes en América Latina y
El Caribe: Un motivo de preocupación. Washington: Organización Panamericana de la Salud. 2008.

50. Anzola M, Peña JP. Metas globales de la Organización Mundial de la Salud para mejorar la nutrición materna, del lactante y del niño pequeño. An Venez Nutr 2014;27(1):26-30. 